\title{
Incidence of Her2/neu receptor positive results in breast cancer cases in
}

\section{Al-Yarmouk teaching hospital}

\section{By}

\author{
Dr. Maitham Mohammed AL-Khateeb ${ }^{1}$; Dr. Ahmed Rabee Abid ${ }^{* 2}$; Dr.Arkan Karrem Abd ${ }^{* 3}$ \\ ${ }^{* 1}$ FACS - C.A.B.S - DGS, Professor General Surgery AL Mustansiriyah Medical College \\ /Consultant surgeon, AL-Yarmouk teaching hospital ; ${ }^{* 2}$ M.B.Ch.B. AL-Yarmouk teaching \\ hospital; ${ }^{* 3}$ Board specialist general surgeon (F.I.M.C.S), AL-Yarmouk teaching hospital
}

\begin{abstract}
:
Back ground: Breast cancer is the most commonly diagnosed cancer in women and the second leading cause of cancer-related death in women. HER2 is a transmembrane receptor with tyrosine kinase. It belongs to a family of four receptors (EGFR/HER1, HER2, HER3, HER4) that are involved in regulating cell growth, survival and differentiation. Structural studies revealed that HER2 is always in an active conformation and ready to interact with the ligand-activated HER receptors, before the advent of HER2-directed therapies, this increased level of HER2 was associated with high recurrence rates and increased mortality in patients with node-positive and node-negative disease.Aim of study: To calculate the incidence of human epidermal growth factor receptor 2 positive results in addition to its relation to estrogen receptor and progesterone receptor results in breast cancer cases in AL-Yarmouk teaching hospital and compare our results to regional and international results. Patients and Methods: Analysis of a prospectively collected clinical database was performed. We included 40 patients who had mastectomy for stage one to stage three breast cancer cases, from October 2018 to October 2019, where by complete receptor information were done. Fluorescence in Situ Hybridization test had been requested. The corresponding H\&E-stained slides from all cases were reviewed. Unstained tissue sections containing tumor, as well as adjacent normal breast parenchyma when possible and corresponding to those used for the Fluorescence In Situ Hybridization test were selected from each case for immunohistochemical staining, comparison with regional and international results was done.Results: Total number of cases collected was 40 cases of breast cancer; they were investigated for human epidermal growth factor receptors 2 in addition to estrogen and progesterone receptors. Age of the patients ranged from 30 to 69 years (mean, 49.27 years). HER2/ neu positive cases were (8) (20\%) of cases, and negative cases were (32) (80\%), in addition to a statically significant relation to ER and PR receptor results. Conclusion: The incidence of human epidermal growth factor receptors 2 positive at AL-Yarmouk teaching hospital was 20\%, estrogen
\end{abstract}


receptors $\backslash$ progesterone receptors positive was (57.5\%) and the relation between these receptors was statistically significant.

Key words: Breast cancer receptors HER2/neu, ER and PR.

\section{Introduction:}

Breast cancer is the most commonly diagnosed cancer in women and the second leading cause of cancer-related death in women ${ }^{(1)}$.Over the last few decades there have been outstanding advances in breast cancer management leading to earlier detection of disease and the development of more effective treatments resulting in significant declines in breast cancer deaths and improved outcomes for women living with the disease.

Breast cancer is no longer seen as a single disease but rather a multifaceted disease comprised of distinct biological subtypes with diverse natural history, presenting a varied spectrum of clinical, pathologic and molecular features with different prognostic and therapeutic implications.Consensus regarding the definitive prognostic/predictive analysis has yet to be reached, but significant progress continues to be made in the ongoing search for a specific, rigorous and reproducible method of identifying successful treatment algorithms utilizing biological markers. $^{(2 ; 3)}$

Breast cancer is a diverse disease with different histological tumor subtypes that can be further characterized on the basis of specific markers. The most commonly examined immunohistochemical markers are estrogen receptor (ER), progesterone receptor (PR), and human epidermal group factor receptor 2 (HER2) ${ }^{(4)}$.

HER2 is a tyrosine kinase member of the epidermal growth factor receptor (EGFR) family and HER2 gene is located on the long arm of chromosome 17 (17q12-21.32). HER2protein is activated upon receptor dimerization that causes autophosphorylation and subsequent downstream signaling ${ }^{(5 ; 6)}$. HER2 positive status of breast cancer patients is assessed by the HER2 protein over expression or HER2 gene amplification. HER2 protein expression is examined using bright field HER2 immunohistochemistry (IHC) with 3,30diaminobenzidine (DAB) detection and HER2 gene level is evaluated by HER2 in situ hybridization (ISH) methods, utilizing various detection approaches such as dual color fluorescent ISH (FISH), chromogenic ISH (CISH), silver ISH (SISH), and dual color ISH (DISH) ${ }^{(7 ; 8)}$. Recently, the gene-protein assay (GPA) has been introduced as a new method for simultaneous evaluation of HER2 IHC and HER2 DISH assays in a single tissue section, allowing pathologists to examine both HER2 protein and HER2 gene statuses simultaneously at the single cell level. HER2 intra tumoral heterogeneity in breast cancer 
can be detected effectively by the concurrent observations of HER2 IHC and DISH results in breast cancer ${ }^{(9)}$.

Over expression of HER2 occurs in approximately $15 \%$ to $20 \%$ of breast cancer and associated with poor prognosis ${ }^{(10)}$.

A HER2-positive cancer cell has approximately two million HER2 proteins on its surface, around 100 times more than a normal cell. ${ }^{(11)}$ This HER2 overexpression causes cells to grow and divide more rapidly. Pairing of HER proteins (also called dimerization) is a vital step in the signaling pathway that leads to cancer cell growth. Signals to encourage the cancer cell to grow and multiply.HER2 homo dimerization or hetero dimerization with other HER family members leads to the activation of downstream signaling pathways that regulate cell growth and survival ${ }^{(12)}$ so In the past, patients with HER2-positive breast cancer were expected to have worse survival outcomes than people with HER2-negative disease. Over the past 15 years, significant progress has been made, and today people with HER2-positive breast cancer treated with HER2-targeted medicines now typically experience better outcomes than people with HER2-negative disease. ${ }^{(13)}$ There is currently no cure for advanced HER2-positive breast cancer. Despite improvements in patient outcomes achieved in recent years, 50 percent of people with advanced disease who are treated with the current standard of care will see their disease progress within 18 months. ${ }^{\text {(14) }}$ Additional treatment options are, therefore, urgently needed for people with advanced HER2positive breast cancer. ${ }^{(15)}$

The humanized trastuzumab binds subdomain IV of the HER2 extracellular domain (ECD). Reported mechanisms of action of trastuzumab include inhibition of HER2 ECD shedding, disruption of downstream signal transduction and mediation of antibody-dependent cell-mediated cytotoxicity ${ }^{(16)}$. Trastuzumab improves clinical outcomes when used with chemotherapy in patients with HER2-positive breast cancer ${ }^{(17 ; 18)}$.

Expression of the estrogen receptor (ER), progesterone receptor (PR) and Her2/neu conventionally determines the therapeutic response and general disease prognosis of primary breast cancer ${ }^{(19 ; 20)}$. Both ER and PR receptors are expressed in approximately $80 \%$ of breast hormone-sensitive tumors and associated with a more favorable prognosis and are followed up with endocrine anti-tumor treatments, such as tamoxifen and aromatase inhibitors, which target the ER.

Tumors with over expressed Her2 are targeted by trastuzumab, which blocks the human epidermal growth factor receptor. In contrast, basal like-breast cancer expresses none of the three breast cancer markers (ER, PR and Her2) and is associated with a poorer prognosis since it is treated with conventional and moderately successful chemotherapies.

Aim of study: To calculate the incidence of human epidermal growth factor receptor 2 positive 
results in addition to its relation to estrogen receptor and progesterone receptor results in breast cancer cases in AL-Yarmouk teaching hospital.

\section{Patients and methods:}

A prospective study which was done at AL-Yarmouk teaching hospital where by 40 cases of modified radical mastectomies were enrolled for the period from October 2019 to October 2020, the cases were confirmed by the triple assessment in breast clinic and excisional biopsy operations, all the samples were sent to a single laboratory and examined by the same histopathologist and the results collected and analyzed according to the age, stage, grade, and HER2 Ineu, ER and PR receptors results.

Case Selection the study material consisted of specimens from 40 consecutive cases of invasive breast carcinoma diagnosed at or referred to AL Yarmouk teaching hospital (breast clinic) in which archival material was available and the Fluorescence In Situ Hybridization test had been requested. The corresponding H\&E-stained slides from all cases were reviewed. Unstained tissue sections containing tumor, as well as adjacent normal breast parenchyma when possible and corresponding to those used for the FISH test, were selected from each case for immunohistochemical staining.

The slides were evaluated for HER-2/neu gene copy number using an epifluorescence microscope.The immunoperoxidase staining was done. Over expression of HER-2/neu was defined as positive membranous staining in more than $10 \%$ of the neoplastic cells. Partial or incomplete, weak to moderate, and moderate to strong membranous staining in more than $10 \%$ of the tumor cells were scored as $1+$ (negative), $2+$ (weak positive), and $3+$ (strong positive), respectively. Borderline cases $2+$ were considered to be equivocal or indeterminate.

All enrolled cases were females, no exclusion criteria.

Statistical analysis was done $\mathrm{P}$ value was calculated by Chi square test was used to test statistical significance. P. value< 0.05 was considered statistically significant. SPSS version 23 was used for statistical analysis.

\section{Results:}

The age of the patients at diagnosis ranged from 30 to 69 years (mean, 49.27 years) and the stander deviation was (18.7) . HER2/ neu positive results were (8), (20\%) of total cases and negative results were (32),(80\%) of total cases and the P-value was 0.7 as shown in (table 1).

Table (1): Distribution of HER2/neu (+ve\&-ve) results relative to the age of patient: 


\begin{tabular}{|c|c|c|c|}
\hline Age $($ years $)$ & HER2 $\$ Heu+ve & HER2 Ineu-ve & Total \\
\hline $30-39$ & $0(0 \%)$ & $3(7.5 \%)$ & $\mathbf{3}(7.5 \%)$ \\
\hline $40-49$ & $3(7.5 \%)$ & $9(22.5 \%)$ & $12(30 \%)$ \\
\hline $50-59$ & $3(7.5 \%)$ & $14(35 \%)$ & $17(42.5 \%)$ \\
\hline $60-69$ & $2(5 \%)$ & $6(15 \%)$ & $\mathbf{8}(20 \%)$ \\
\hline total & $\mathbf{8}(20 \%)$ & $32(80 \%)$ & $40(100 \%)$ \\
\hline
\end{tabular}

There is no significant statistical relationship between the age and human epidermal growth factor positivity.

Disease stage according to TNM staging system varied from stage (0) to III , stage 0 was 1 case, stage 1 was 19 cases, stage 11 was 18 cases and stage 111 was 2 cases, P.value 0.1 and the distribution of HER2/neu receptors according to the disease stages shown in the( table 2).

Table (2): Distribution of HER2/neu results relative to the stage of the disease:

\begin{tabular}{|c|c|c|c|}
\hline Stage & HER2\neu+ve & HER2\neu-ve & Total \\
\hline 0 & $1(2.5 \%)$ & $0(0 \%)$ & $1(2.5 \%)$ \\
\hline I & $1(2.5 \%)$ & $18(45 \%)$ & $19(47.5 \%)$ \\
\hline II & $5(12.5 \%)$ & $13(32.5 \%)$ & $18(45 \%)$ \\
\hline III & $1(2.5 \%)$ & $1(2.5 \%)$ & $2(5 \%)$ \\
\hline Total & $\mathbf{8}(20 \%)$ & $32(80 \%)$ & $40(100 \%)$ \\
\hline
\end{tabular}

There is no significant statistical relationship between the TNM stage and human epidermal growth factor positivity.

Disease grade varied from grade I to III, grade 1 was 2 negative cases, grade 11 was 22 cases and grade III was 16 cases, P.value 0.3 and the distribution of HER2/neu receptors according to the disease grade is shown in the (table 3 ).

Table (3): Distribution of HER2/neu results relative to the grade of the disease:

\begin{tabular}{|c|c|c|c|}
\hline Grade & HER2\neu+ve & HER2\neu-ve & Total \\
\hline I & $0(0 \%)$ & $2(5 \%)$ & $2(5 \%)$ \\
\hline II & $\mathbf{3 ( 7 . 5 \% )}$ & $19(47.5 \%)$ & $22(55 \%)$ \\
\hline III & $5(12.5 \%)$ & $11(27.5 \%)$ & $16(40 \%)$ \\
\hline total & $\mathbf{8}(20 \%)$ & $32(80 \%)$ & $\mathbf{4 0}(100 \%)$ \\
\hline
\end{tabular}


There is no significant statistical relationship between the grade of cancer and human epidermal growth factor positivity.

Estrogen and progesterone receptors results were investigated relative to HER2/neu results and ER/PR+ve results was 23 cases and ER/PR-ve was 17 cases, P-value (0.03) and the distribution of ER/PR receptors relative to the HER2/neu were shown in (table4):

Table (4): Distribution of HER2/neu results relative to ER/PR receptors results:

\begin{tabular}{|c|c|c|c|}
\hline & HER2\neu+ve & HER2\neu-ve & Total \\
\hline ER\PR+ve & $2(5 \%)$ & $21(52.5 \%)$ & $23(57.5 \%)$ \\
\hline ER\PR-ve & $6(15 \%)$ & $11(27.5 \%)$ & $17(42.5 \%)$ \\
\hline Total & $8(20 \%)$ & $32(80 \%)$ & $40(100 \%)$ \\
\hline
\end{tabular}

There is a significant relationship between the estrogen receptor \&progesterone receptor and human epidermal growth factor result.

\section{Discussion:}

It is well established that 15-20\% of breast cancer patients are HER2 positive and it is associated with poor prognosis of breast cancer patients. After the discovery of HER2 gene, the significance of HER2 gene role was demonstrated by the correlation of overall survival and time to relapse with the HER2 gene amplification in breast cancer. Additionally, the association of the HER2 gene implications with the HER2 protein over expression was proved by immunohistochemical and Western blotting analyses ${ }^{(21)}$

Regarding the age of the patients at diagnosis we found that it ranged from 30 to 69 years (mean, 49.27 years). HER2/ neu +ve were (8) (20\%) cases and -ve were (32) (80\%), In another study by Adedayo et al.,2008 in Marshfield Clinic Weston Center, they found Final analysis included 1134 invasive breast cancer subjects identified in the Marshfield Clinic/St. Joseph's Hospital Cancer Registry from January 1, 1998 to June 30, 2005. The mean age of all subjects was 62.7 years. $116(10.2 \%)$ were ER/PR+, Her2+, 781 (68.9\%) were ER/PR+,Her2-, 85 (7.5\%) were ER/PR-,Her2+, and the remaining $152(13.4 \%)$ were classified as triple negative ${ }^{(22)}$

Disease stage varied from stage (0) to (3), stage 0 was 1 case (2.5\%), stage 1 was 19 cases (47.5\%), stage II was 18 cases (45\%) and stage III was 2 cases (5\%), In study by Azizun-Nisa et al., 2008.Stratification of tumor size was performed in 3 groups, group 1(tumorsize $\leq 2 \mathrm{~cm}$ ), group 2 $(2-5 \mathrm{cms})$ and group $3(\geq 5 \mathrm{cms})$ in diameter.

The total number of group 1 tumors was 18 [9 (50\%) ER positive; 8 (44.44\%) each PR and Her-2 positive]. Of the group 2 tumors 26 (32.91\%) were ER positive, 21 (26.58\%) were PR positive and 
$27(34.18 \%)$ were HER-2 positive. Of the group 3 tumors 14 (26.42\%) were ER positive, 9 (16.98\%) were PR positive and 21 (39.62\%) were HER-2 positive. ER expression in the HER$2 /$ neu positive, large sized tumors was significantly decreased compared with smaller tumors (ER $6.3 \%$ vs. 11.8$)^{(23)}$.

Regarding the disease grade the results varied from grade (1) to (3), grade 1 was 2 cases (5\%), grade II was 22 cases (55\%) and grade III was 16 cases (40\%), While in study by Azizun-Nisa et al., 2008. the mean age of the patients was 48.3. The predominant morphology was infiltrating ductal carcinoma (85.3\%). The majority of the cases presented as grade II (55.3\%) lesions with tumor necrosis (70\%) and lymph node involvement (71.3\%). ER and PR were positive in $32.7 \%$ and $25.3 \%$ cases respectively. HER-2/neu was positive $(3+)$ in24.7. ${ }^{(23)}$

Estrogen and progesterone receptors positivity was investigated and ER/PR+ve was 23 cases $(57.5 \%)$ and ER/PR-ve was 17 cases $(42.5 \%)$, and the relation between ER\PR receptors and HER2\neu receptors was significant (P. value (0.03) Azizun- Nisa et al., 2008. found ER positivity was observed in $70 \%$ grade I, $48.2 \%$ grade II and $3.5 \%$ grade III carcinomas (p value $<0.001$ ). Similarly PR positivity was observed in $70 \%$ grade I, $36.14 \%$ grade II and $1.75 \%$ grade III carcinomas (p value $<0.001)$. HER-2 was positive in $1(10 \%)$ case of grade I carcinoma, 31 $(37.35 \%)$ cases of the grade II carcinoma and $24(42.11 \%)$ cases of grade III carcinoma. In the HER-2/neu positive tumors, ER and PR expression in high grade tumors was significantly decreased compared with intermediate grade tumors (ER 5.6\% vs. 10.5; PR 0\% vs5.3\%). ${ }^{(23)}$

In study by Lajos Pusztai et al., 2008 they found Two hundred five patients (23\%) were designated as having TNBC and 863 patients (77\%) were designated as non- TNBC. Expression of ER, PR, orHER-2 was observed in 645(58\%), 524(47\%), and 272 (24\%) patients, respectively. The mean age of patients with TNBC (48years) was slightly younger compared with non-TNBC (50 years; P.002). ${ }^{(24)}$

\section{Conclusion:}

The incidence of human epidermal growth factor receptors 2 positive results at AL-Yarmouk teaching hospital was $20 \%$, estrogen receptors \progesterone receptors positive was $(57.5 \%)$ and the relation between these receptors was significant and the HER2neu incidence has no statistically significant association with age or grade or stage of the disease.

\section{References:}

1 .

Ferlay J, Shin HR, Bray F, Forman D, Mathers C, Parkin DM (2010)

Estimates of worldwide burden of cancer in 2008: GLOBOCAN 2008. Int J Cancer127:28932917 
2.

Glass AG, Lacey JV Jr, Carreon JD, Hoover RN. Breast cancer incidence, 1980-2006: combined roles of menopausal hormone therapy, screening mammography, and estrogen receptor status. J Natl Cancer Inst2007; 99:1152-1161.

3.

Ravdin PM, Cronin KA, Howlader N, Berg CD, Chlebowski RT, Feuer EJ,

Edwards BK, Berry DA. The decrease in breast cancer incidence in 2003 in the United States. N Engl J Med 2007;356:1670-1674

4. Bertos NR, Park M (2011) Breast cancer-one term, many entities? J Clin Invest121:3789-3796

5.

Citri A, Yarden Y (2006) EGF-ERBB signalling: towards the systems level. Nat Rev Mol Cell Biol 7:505-516

6.

Graus-Porta D, Beerli RR, Daly JM, Hynes NE (1997) ErbB-2, the preferred heterodimerization partner of all ErbB receptors, is a mediator of lateral signaling. EMBO J16:1647-1655

7.

Francis GD, Jones MA, Beadle GF, Stein SR (2009) Bright-field in situ hybridization for HER2 gene amplification in breast cancer using tissue microarrays: correlation between chromogenic (CISH) and automated silver-enhanced (SISH) methods with patient outcome. DiagnMolPathol18:88-95

8.

Nitta H, Hauss-Wegrzyniak B, Lehrkamp M, Murillo AE, Gaire F, Farrell M et al (2008) Development of automated brightfield double in situ hybridization (BDISH) application for HER2 gene and chromosome 17 centromere (CEN 17) for breast carcinomas and an assay performance comparison to manual dual color HER2 fluorescence in situ hybridization (FISH). DiagnPathol3:41

9.

Nitta H, Kelly BD, Padilla M, Wick N, Brunhoeber P, Bai I et al (2012) A gene-protein assay for human epidermal growth factor receptor 2 (HER2): brightfield tricolor visualization of HER2 protein, the HER2 gene, and chromosome 17 centromere (CEN17) in formalin-fixed, paraffin-embedded breast cancer tissue sections. DiagnPathol7:60

Slamon DJ, Clark GM, Wong SG, Levin WJ, Ullrich A, McGuire WL. Human breast cancer: correlation of relapse and survival with Amplification of the HER2/neuoncogene. Science 1987; 235: 177- 82.

11. . Lewis Phillips GD, et al. Cancer Res2008;68(22):9280-90.

12. YardenY, SliwkowskiMX.UntanglingtheErbBsignallingnetwork.NatRevMol Cell Biol2001; 2:127-37.

13.

Dawood S. et al., J ClinOncol2010;28(1):92-8.

14. Baselga J, et al. N Engl J Med2012;366(2):109-19. 
15.

Nahta R. et al., Nat ClinPract 2006;3(5):269-80.

16.

Spector NL, Blackwell KL. Understanding the mechanisms behind trastuzumab therapy for human epidermal growth factor 2- positive breast cancer. J ClinOncol2009; 27:5838-47.

17.

Mariani G, Fasolo A, De Benedictis E, Gianni L. Trastuzumab as adjuvant systemic therapy for HER2-positive breast cancer. Nat ClinPractOncol2009; 6:93-104.

Del Mastro L, Lambertini M, Bighin C, Levaggi a, D'Alonzo a, Giraudi S, et al. Trastuzumab as first-line therapy in HER2-positive metastatic breast cancer patients. Expert Rev Anticancer Ther 2012; 12:1391-405.

19.

Janssen EA, Slewa A, Gudlaugsson E, Jonsdottir K, Skaland I, Søiland H, et al. Biologic profiling of lymph node negative breast cancers by means of microRNA expression. Mod Pathol 23:1567- 76.

Bertheau P, Turpin E, Rickman DS, Espie M, de Reynies A, Feugeas JP, et al. Exquisite sensitivity of Tp53 mutant and basal breast cancers to a dose-dense epirubicincyclophosphamide regimen. PLoS Med 2007;4:90.

21.

Salmon DJ, Clark GM, Wong SG, Levin WJ, Ullrich A, McGuire WL (1987)

Human breast cancer: correlation of relapse and survival with amplification of the HER-2/neu oncogene. Science 235:177- 182

Adebayo A. Onitilo, MD, MSCR, FACP, Marshfield Clinic Weston Center, 3501 Cranberry Boulevard, Weston, WI 54476, Tel: 715- 393-1400, IL, May 30-June 3,2008.

Pathology-based Cancer Registry, Dept. of Pathology \& Microbiology , Aga Khan University Hospital, Stadium Road P.O. Box 3500 , Karachi 74800, Pakistan Email: bhurgri@ cyber.net.pk, Asian Pacific Journal of Cancer Prevention, Vol 9,2008

Lajos Pusztai, MD, PhD, Department of Breast Medical Oncology, Unit 1354, The University of Texas M.D. Anderson Cancer Center, 1515 Holcombe Blvd, Houston, TX 77030-1439, USA; e-mail: lpusztai@ mdanderson.org.@ 2008 by American Society of Clinical Oncology. 